\title{
Some Problems of Surgery in a Leprosy Colony.
}

A. Roy.

$7 \mathrm{HE}$ surgical aspect of the treatment of leprosy has not received much attention hitherto. The reasons for this are firstly, because this aspect of leprosy has practically nothing to do with its curative treatment. In this connection the only thing that has been commonly done is nerve stretching in the earlier neural cases, and this operation is done not necessarily with a view to treating leprosy, but in order that the nerve pain may be reduced and as an attempt to avoid later contractures to fingers and toes.

Secondly, the incentive for taking up surgical work in such cases is not strong, because a surgeon operates in order that he may relieve the patient's suffering and rid him of his complaint. In the case of those suffering from leprosy, surgical operations are often only palliative, and in many cases, such as the amputation of the leg, the patient's incapacity may be greater, not only because he has lost his limb, but the other 'leg may become worse owing to the greater strain placed on it. Further, in operating on those with crippled hands, the surgeon may prolong the patient's life, but in doing so he may increase his suffering, and one has to consider carefully such cases before operating, lest, as a result of the operation, incapacity may be actually increased. However, in many cases suffering is quite definitely prevented.

Thirdly, the results of operations are not always encouraging because few seem to heal up by first attention, even with the most asceptic care.

My plea for writing this note is mainly to show the difference not only in the results but also in the methods of dealing with the surgical aspect of the treatment. I have also noted that many doctors who have visited our colony mention their own methods of general treatment which are applicable to ordinary cases, but it is hard to convince them that general surgical methods are not always applicable to those suffering from leprosy. I shall now illustrate my remarks by referring to the following operations :-

Amputations.-These are the most common operations here. The incidence of cases which need amputation increases after the winter season. 
Minor operations, (toes and fingers).-When there are ulcers on the tips of the toes and fingers a general practitioner may wonder why these tiny ulcers do not heal easily. When I was treating these cases in the general way I found a small percentage of cases did heal up though they took a longer time; but they were almost sure to break out again within a short period. Other cases did not heal up without snipping the tips off, but even some of these cases recurred after a longer period. I next started to disarticulate these bones at the nearest healthy joint. These efforts were more successful. Patients realised that as a result they no longer needed to visit the hospital daily for months together.

Trophic Ulcers.-In these cases I used to incise the tissues along the bone, snip off as far as I could, and at the same time cut off the tendens attached to the bone, pulling them out as far as possible. This method helped the patients, but the results were not permanent. Some returned for treatment after the next winter or even before that. Now we have discarded this method totally and instead dissect out the bone, which, though it appeared at first a barbarous procedure, is to my mind the best method of dealing with these cases. "There is only a tiny ulcer with slight necrosis of bone; what is the necessity of performing such big operations?" This was the complaint made by many of the sufferers, and even by some of our colleagues. But now our experience has shown us, after dealing with no less than 150 cases, the excellent permanent results obtained. We cannot but say "it is the method of choice."

\section{Description OF TECHNiQUe.}

Dr. Cochrane had conceived the idea of dissecting out the affected bones some years ago. I saw him trying this method in 1927. But he did not then give it an extensive trial probably for want of proper facilities. This time, owing to the able assistance and help of Mrs. Miller and the hospital staff at Purulia, all the beds in the hospital were filled up with such cases. The technique was as follows:-

Make an incision at the distal end of the necrosed bone on the dorsum of the foot or hand. Deepen the incision until the bone is reached, then extend it along the bone right up to its joint. Endeavour not to expose and damage the bone behind. Then disarticulate the joint carefully and dissect out the necrosed bone. Twisting the joint with lion forceps at the last stage makes the separation of the 
bone easier. As far as possible clean up the ragged edges of the wound and snip away dead tissue, packing the wound with gauze soaked in 1-1000 acriflavine. One or two stitches may be inserted at the proximal end of the wound in order to cover the joint if exposed. Dress daily with acriflavine, but if there is sloughing change to eusol twice daily, or more often according to the requirements of the case. Wounds take on an average three weeks to heal up. The following points need emphasising :-

(1) Make a bold, clean incision.

(2) Try not to expose the next bone behind ; if exposed stitch the skin over it to cover.

(3) Deep sutures are of no use.

(4) Never try to do anything on the sole of the foot, or the palm of the hand.

(5) On opening the case the next day the edges may be found necrosed, probably due to repeated incisions in exposing the joint. Simple dressing with eusol will clear up this condition.

The advantages of this method are that the patients are saved daily visits to the hospital for dressing for a long period. They are also saved from the risk of sepsis, which in some cases may necessitate an amputation. Though it is troublesome and expensive in the beginning, it is more economical in the long run.

The disadvantages are that whatever precautions are taken the scalpel is sure to be useless for the next case. This may be avoided by using old scalpels to disarticulate the necrosed bone.

Lately we have improved the above technique to some extent; on taking out the bone we plug the wound with acriflavine gauze as usual, but stitch the skin above. We have named these stitches " 24 hours' stitches," because we take out the stitches the next day. The object of putting them in is to prevent the wound from gaping too much. The big gap made by the tight plugging takes longer to granulate up.

\section{Major Amputation of Limbs.}

After amputating the limbs it is never safe to stitch from end to end. The patients have no vitality to absorb the oozing which accumulates beneath the stitches. A drainage tube is too irritating to the tissues. We have therefore found that a piece of fine gauze rolled up with a piece of fine rubber (a portion of torn glove) answers quite well. 
Syme's amputation (an amputation at the ankle joint by a heel flap, with removal of malleoli) and Pirogoff's amputation (an amputation at the ankle joints in which the posterior part of the oscalsis is retained and united to the sawn surface of the tibia) are never advisable for cases of leprosy, where all the metatarsals are bad. In these cases amputation of the limb at the lower third is advisable. We tried these methods on two cases where all the metatarsals were necrosed. They still visit the hospital claily for dressing.

Cataracts.-We have not yet been able to find out any new method of operative technique for these, but we are sure that any lack of absolute rest for a fairly long period after the operation undoes the whole effort of operation. Before deciding to operate on cataract in a case of leprosy, it is necessary to make sure that the ultimate result of the operation would not be nullified by secondary infection lurking in the conjunctiva or in the lachrymal sacs and ducts, as in these places, especially in leprotic cases, rich bacterial flora almost always exist. A thorough cleaning of the conjunctiva, and rest to the eye for at least three or four days, should be an essential preliminary to any operative treatment on the eye. Strong antiseptics should never be used for cleaning the eyes, as the conjunctiva and the sclera in cases of leprosy are, in the majority of cases, devitalised already by the involvement of the ophthalmic branch of the fifth nerve, and there is usually superficial anæsthesia of the cornea with resultant loss of the protective influence exerted by the corneal reflex. Involvement of the other nerves supplying the orbital structures, as for example the supra-orbital, the orbital branches of the facial, indirectly influence the vitality of the conjunctiva and the sclera. The dirty nasal cavity in the vicinity of the eye may some time spoil the chance of recovery after operation by ascending the sepsis from the nasal cavity through the nasolachrymal duct.

The occurrence of ectropion in the late neural stages of the disease marks the crowning tragedy of leprosy, as this inevitably leads on to corneal ulcers resulting in leucoma and loss or diminution of vision of varying degrees. Palliative measures usually advocated in similar cases in non-leprotic cases, as for example, wearing a shade, frequent antiseptic washings and smearing of the eyelids at bed time with liquid paraffin, etc., prove unavailing in cases of leprosy, as in the latter there are several other factors that complicate the ectropion. By the time the ectropion is detected in a case 
of leprosy there is usually some degree of anæsthesia of the cornea and devitalisation of the conjunctiva and sclera. As a result of loss of corneal reflex there is already some latent infection of the conjunctiva and some injury to the cornea. In these cases palliative measures are worse than useless.

Application of paraffin to the eyelids of these cases has been known to afford a shelter to the secondary organisms which were enabled to multiply in the canthi and in the fornices of the conjunctiva, and produce a purulent exudation.

After a visit from Dr. Gass, of Chandkuri, he demonstrated an operation on lateral tarsorrophy. We continued to perform this operation, and have found since then that great care has to be taken in the selection of cases. A cripple patient cannot be expected to keep his eyes clean after the operation. If the eye is not kept clean, the artificial protection afforded to the bulber conjunctiva and the cornea by the operation will form a pocket in which secondary organisms can multiply vigorously and make the result of operation worse than the original ectropion itself. We have had experience of this lately, and therefore, in crippled cases one should hesitate before attempting a canthorraphy.

Finally, to secure uniformly successful results after operation it is essential to examine the stools for hookworm ova and erradicate them if they exist, otherwise wounds do not heal up quickly owing to the paucity of hœmoglobin.

Acknowledgments are due to Mrs. Miller, Dr. Rao, and to Miss Thornton, for their kind help and guidance, and also to Mr. A. D. Miller for supplying the photos. 